\title{
On the sources of the height-intelligence correlation: New insights from a bivariate ACE model with assortative mating
}

\author{
Jonathan P. Beauchamp · David Cesarini • \\ Magnus Johannesson • Erik Lindqvist • \\ Coren Apicella
}

Received: 30 December 2009/ Accepted: 14 June 2010/Published online: 6 July 2010

(C) The Author(s) 2010. This article is published with open access at Springerlink.com

\begin{abstract}
A robust positive correlation between height and intelligence, as measured by IQ tests, has been established in the literature. This paper makes several contributions toward establishing the causes of this association. First, we extend the standard bivariate ACE model to account for assortative mating. The more general theoretical framework provides several key insights, including formulas to decompose a crosstrait genetic correlation into components attributable to assortative mating and pleiotropy and to decompose a crosstrait within-family correlation. Second, we use a large dataset of male twins drawn from Swedish conscription records and examine how well genetic and environmental factors explain the association between (i) height and intelligence and (ii) height and military aptitude, a professional psychogologist's assessment of a conscript's ability to deal with wartime stress.
\end{abstract}

Edited by Stacey Cherny.

J. P. Beauchamp $(\bowtie)$

Department of Economics, Harvard University, Cambridge, MA 02138, USA

e-mail: jpbeauch@fas.harvard.edu

D. Cesarini

Department of Economics, New York University, New York, NY 10012, USA

M. Johannesson

Department of Economics, Stockholm School of Economics, Box 6501, Stockholm 113 83, Sweden

E. Lindqvist

Research Institute of Industrial Economics, P.O. Box 55665, Stockholm 102 15, Sweden

C. Apicella

Department of Health Care Policy, Harvard Medical School, Boston, MA 02115, USA
For both traits, we find suggestive evidence of a shared genetic architecture with height, but we demonstrate that point estimates are very sensitive to assumed degrees of assortative mating. Third, we report a significant within-family correlation between height and intelligence $(\hat{\rho}=0.10)$, suggesting that pleiotropy might be at play.

Keywords Assortative mating - Bivariate ACE model Genetic correlation · Height · Intelligence · IQ ·

Within-family correlation

\section{Introduction}

A robust positive correlation between height and intelligence, as measured by IQ tests, ${ }^{1}$ has been established in the literature with little consensus regarding its cause (Humphreys et al. 1985; Johnson 1991; Kanazawa and Reyniers 2009; Tanner 1979; Teasdale et al. 1989; Wheeler et al. 2004). Both environmental and genetic explanations have been advanced. For instance, previous research has found that markers of prenatal quality and nutritional status during childhood are associated with height (Eide et al. 2005; Steckel 1995) and cognition in adulthood (See GomezPinilla 2008; Martyn et al. 1996; Seidman et al. 1992; Sørensen et al. 1997), suggesting that early environmental factors may be responsible for the correlation between height and intelligence (Abbott et al. 1998). Other evidence in support of environmental channels is the decline in the correlation between height and intelligence over time observed in the Scandinavian countries (Teasdale et al. 1989; Sundet et al. 2005; Tuvemo et al. 1999). On the other hand, reported

\footnotetext{
${ }^{1}$ We follow the literature and use the term "intelligence" to refer to what those IQ tests measure.
} 
heritability estimates in adults for measures of intelligence (e.g. 75\%) (Neisser et al. 1996) and height (e.g. 87-93\%) (Silventoinen et al. 2003) are high, suggesting that the relationship may instead be genetically mediated. For example, growth hormone deficiency, which is sometimes caused by genetic mutations, is characterized by both short stature and cognitive impairments (van Dam et al. 2005). Evidence of a shared genetic architecture between brain volume, intracranial space, and height (Posthuma et al. 2000) may also be interpreted as evidence of genetic mediation of the height-intelligence correlation.

Two studies, using twin data, have attempted to estimate the components of the height-intelligence correlation that are due to genetic and environmental effects. The results were mixed. Silventoinen et al. (2006) found in several samples of Dutch twins that the association between height and intelligence is primarily genetic in origin. Sundet et al. (2005), using conscription data from a considerably larger and more representative sample of Norwegian twins, found that the association between height and intelligence is primarily explained by common environmental factors. Both papers use a standard bivariate ACE model to arrive at these conclusions.

The present study makes several contributions toward elucidating the sources of covariation between height and intelligence. First, we extend the bivariate ACE model to allow for assortative mating and derive a general formula for decomposing a cross-trait genetic correlation into components attributable to assortative mating and pleiotropy. This provides a clear theoretical framework for studying the correlation between two traits and is particularly useful when examining phenotypes for which there is high assortative mating, height and intelligence being prime examples. The model demonstrates how sensitive estimates from the bivariate ACE model are to assumptions about assortative mating. Second, we use a sample of male Swedish twins matched to conscription records to examine the relative importance of genetic and environmental factors in explaining the association between height and intelligence. The sample used is the largest to date for such a study. It includes an additional measure of cognitive function other than intelligence, measured during enlistment through interviews by a military psychologist. This measure, which we label military aptitude, has a strong predictive validity for labor market outcomes independent of intelligence, such as wages, earnings and unemployment (Lindqvist and Vestman forthcoming). We apply the standard bivariate ACE model to decompose the height-intelligence and the height-military aptitude correlations, but we caution that the resulting estimates are very sensitive to assumptions about assortative mating and illustrate the sensitivity of these estimates by reporting results for different assumed levels of assortative mating. Lastly, we report a significant within-family heightintelligence correlation $(\hat{\rho}=0.10)$, consistent with the hypothesis that pleiotropy (or linkage) accounts for part of the genetic correlation.

\section{Method}

Sample

Our data comes from two main sources: the Swedish Twin Registry (STR) and the Swedish National Service Administration (SNSA). The STR contains information on nearly all twin births in Sweden since 1886, and has been described in further detail elsewhere (Lichtenstein et al. 2006). The sample includes those individuals who have participated in at least one of the Twin Registry's surveys. The primary datasource is SALT (Screening Across the Lifespan Twin study). This was a survey administered to all Swedish twins born between 1926 and 1958 and attained a response rate of $74 \%$. Fifty percent of the subjects in the dataset are from the SALT cohort. The secondary source is the web-based survey STAGE (The Study of Twin Adults: Genes and Environment). This was a web-based survey administered between November 2005 and March 2006 to all twins born in Sweden between 1959 and 1985. It attained a response rate of $61 \%$. Approximately $30 \%$ of our subjects are drawn from STAGE. Our final datasource comes from a survey sent out in 1973 to the same cohort as SALT (Lichtenstein et al. 2006).

We matched the Swedish twins to the conscription data provided by SNSA. All Swedish men are required by law to participate in a nationwide military conscription at the age of 18. Before 1990, exemptions were very rare. The actual drafting procedure can take several days during which recruits undergo medical and psychological examination. The basic structure of the administered intelligence test has remained unchanged during our study period, though minor changes took place in 1980 and 1994. Recruits take four subtests (logical, verbal, spatial and technical) which, for most of the study period, are graded on a scale from 0 to 40 . These raw scores are converted to a ordinal variable ranging from 1 to 9. Carlstedt (2000) discusses the history of psychometric testing in the Swedish military and provides evidence that this test of intelligence is a good measure of general intelligence. Thus, this test differs from the AFQT, which focuses more on "crystallized" intelligence.

All conscripts also see a psychologist for a structured interview. The psychologist has access to background information on the interviewee, such as school grades, medical background, cognitive ability and answers to a battery of questions on friends, family, and life. In conducting the interview, the psychologist is required to follow a manual and, ultimately, to make an assessment of the prospective recruit's capacity to handle stress in a war situation. In making the assessment, the psychologist considers an individual's ability 
to function in a group, adapt to new environments, as well as his persistance and emotional stability. Motivation for doing the military service is not among the set of characteristics that is considered beneficial for succeeding in the military. The psychologist assigns each interviewee an ordinal score from 1 to 9, but again these are constructed from four raw scores, this time ranging from 1 to 5 . Like the intelligence test score, the military aptitude score is subject to measurement error because of random influences on conscript performance and because conscripts may differ in their motivation for the military service. ${ }^{2}$ Lindqvist and Vestman (forthcoming) provide a more detailed description of the personality measure used by SNSA.

For both intelligence and military aptitude, the raw scores underlying an individual's ordinal score are available to us. The raw scores are percentile rank-transformed and then converted by taking the inverse of the standard normal distribution to produce normally distributed test scores. The transformation is done separately for each year, but when less than 100 pairs are available for a particular year, two adjoint years are pooled. Since the sample of women who enlist in the military comprises a small and highly self-selected group, this paper focuses exclusively on men. We restrict the sample to all male twin pairs for whom complete data on cognitive ability and military aptitude is available for both twins. This leaves 1246 pairs of monozygotic (MZ) twins and 1568 pairs of dizygotic (DZ) twins for analysis, all of which are born between 1950 and 1976. Descriptive statistics for the twins in our sample are presented in Table 1, disaggregated by zygosity. For expositional convenience, we report the ordinal instead of the normalized scores in the Table, even though the latter are used in the analyses that follow.

The bivariate ACE model, assortative mating and the cross-trait genetic correlation

\section{The bivariate ACE model}

We follow biometrical genetic theory (Falconer and Mackay 1981) and previous papers on this subject (Silventoinen et al. 2006; Sundet et al. 2005) to decompose the variance of a phenotype into shares attributable to additive genetic factors, common environment, and individual environment. Our empirical analysis uses the standard bivariate ACE model (Neale and Cardon 1992). Let $Y^{i}=\left(Y_{1}^{i}, Y_{2}^{i}\right)^{\prime}$ be a vector of two observable phenotypes of individual $i$, and suppose that

\footnotetext{
${ }^{2}$ However, the military aptitude score is subject to an additional form of measurement error since psychologists will vary in their judgement of identical conscripts. Lilieblad and Ståhlberg (1977) estimated the correlation between the SNSA psychologists' assessment to be .85 after letting thirty SNSA psychologists listen to tape recordings of thirty enlistment interviews.
}

Table 1 Background variables

\begin{tabular}{llll}
\hline & $\mathrm{MZ}$ & $\mathrm{DZ}$ & Population \\
\hline Income (in SEK) & 342,631 & 335,987 & 325,245 \\
SD & 241,060 & 340,271 & 258,867 \\
Education (years) & 12.50 & 12.14 & 12.54 \\
SD & 2.68 & 2.63 & 2.35 \\
1 if married & 0.50 & 0.51 & 0.51 \\
SD & - & - & - \\
Age in 2005 & 48.85 & 51.69 & 45.43 \\
SD & 7.66 & 6.37 & 3.63 \\
Intelligence & 5.12 & 4.94 & 5.13 \\
SD & 1.88 & 1.94 & 1.94 \\
Military aptitude & 5.32 & 5.17 & 5.08 \\
SD & 1.69 & 1.78 & 1.78 \\
Height (in cm) & 178.45 & 178.60 & 178.94 \\
SD & 6.60 & 6.46 & 6.56 \\
\hline
\end{tabular}

Note: Income (förvärvsinkomst) is defined as the sum of income earned from wage labor, income from own business, pension income and unemployment compensation. Capital income is not included. The education variable produced by Statistics Sweden is categorical (with seven categories ranging from middle school to $\mathrm{PhD}$ ). The categorical scores are converted to years of education using the imputation model of Isacsson (2004). Population data was estimated using information from Statistics Sweden on a representative sample born between 1954 and 1965. In our analyses, we do not use the ordinal scores but normalized scores computed from subscores for intelligence and military aptitude, as described in the text

$Y^{i}=\mathbf{a} A^{i}+\mathbf{c} C^{i}+\mathbf{e} E^{i}$,

where a, $\mathbf{c}$, and $\mathbf{e}$ are $2 \times 2$ matrices of coefficients and where $A^{i}=\left(A_{1}^{i}, A_{2}^{i}\right)^{\prime}, C^{i}=\left(C_{1}^{i}, C_{2}^{i}\right)^{\prime}$ and $E^{i}=\left(E_{1}^{i}, E_{2}^{i}\right)^{\prime}$ are, respectively, the latent additive genetic, common environmental, and individual environmental factors underlying traits $Y_{1}^{i}$ and $Y_{2}^{i}$. Throughout, we make the standard assumption that $A, C$, and $E$ are mutually independent. The model assumes that all genetic variance is additive, thereby ruling out dominance and epistasis. Suppose further, without loss of generality, that all the variables have been standardized to have mean zero and unit variance. The correlation between the traits of two individuals $i$ and $j$ will then be equal to

$\mathbf{E}\left[Y^{i} Y^{j^{\prime}}\right]=\mathbf{a E}\left[A^{i} A^{j \prime}\right] \mathbf{a}^{\prime}+\mathbf{c E}\left[C^{i} C^{j \prime}\right] \mathbf{c}^{\prime}+\mathbf{e E}\left[E^{i} E^{j \prime}\right] \mathbf{e}^{\prime}$,

where $\mathbf{E}$ denotes the expectations operator. It follows from the standardization that the expectation of the product of two variables is simply their correlation. Table 2 summarizes a set of assumptions about $\mathbf{E}\left[A^{i} A^{j^{\prime}}\right], \quad \mathbf{E}\left[C^{i} C^{i^{\prime}}\right]$ and $\mathbf{E}\left[E^{i} E^{j^{\prime}}\right]$. These assumptions are customary in the behavior genetics literature, except for the presence of the $\Gamma_{M}$ matrix which, as we will show, accounts for assortative mating at the additive genetic level. The usual bivariate ACE model does not account for assortative mating and thus implicitly 
Table 2 Assumed values of $\mathbf{E}\left[A^{i} A^{j \prime}\right], \quad \mathbf{E}\left[C^{i} C^{j \prime}\right]$ and $\mathbf{E}\left[E^{i} E^{j \prime}\right]$ for different relationships between individuals $\mathrm{I}$ and $\mathbf{J}$

\begin{tabular}{lllll}
\hline & \multicolumn{4}{l}{ Relationship between individuals $i$ and $j$} \\
\cline { 2 - 5 } & $i=j$ & MZ twins & DZ twins & Unrelated \\
\hline $\mathbf{E}\left[A^{i} A^{j \prime}\right]$ & $\Gamma_{A}$ & $\Gamma_{A}$ & $\frac{1}{2}\left(\Gamma_{A}+\Gamma_{M}\right)$ & 0 \\
$\mathbf{E}\left[C^{i} C^{j \prime}\right]$ & $\Gamma_{C}$ & $\Gamma_{C}$ & $\Gamma_{C}$ & 0 \\
$\mathbf{E}\left[E^{i} E^{j \prime}\right]$ & $\Gamma_{E}$ & 0 & 0 & 0
\end{tabular}

Note: This table shows the assumptions made about $\mathbf{E}\left[A^{i} A^{j \prime}\right]$,

$\mathbf{E}\left[C^{i} C^{j \prime}\right], \mathbf{E}\left[E^{i} E^{j \prime}\right]$ within individuals, between MZ twins, between DZ twins and between unrelated individuals

assumes that $\Gamma_{M}$ is a zero matrix. In the next section of the paper, we show that assortative mating enters the model this way, through $\Gamma_{M}$.

Behavior geneticists have previously studied models which allow for assortative mating, but they were mostly concerned with the case of a single phenotype (see, for instance, Eaves et al. 1978; Eaves and Heath 1981; Martin et al. 1986; Keller et al. 2009). Eaves et al. (1984) consider the multivariate case and develop a model in which there is assortative mating on a latent phenotype. We add to this literature by deriving formulas that describe, among others, the effects of assortative mating on the genetic correlation between two traits and by augmenting the bivariate ACE model to account for these effects.

The $\Gamma$ matrices in Table 2 have the following elements:

$$
\begin{aligned}
\Gamma_{A} & =\left(\begin{array}{cc}
1 & \rho_{A} \\
\rho_{A} & 1
\end{array}\right), \Gamma_{C}=\left(\begin{array}{cc}
1 & \rho_{C} \\
\rho_{C} & 1
\end{array}\right), \Gamma_{E}=\left(\begin{array}{cc}
1 & \rho_{E} \\
\rho_{E} & 1
\end{array}\right) \\
\text { and } \Gamma_{M} & =\left(\begin{array}{ll}
m_{11} & \bar{m}_{12} \\
\bar{m}_{12} & m_{22}
\end{array}\right) .
\end{aligned}
$$

As we show in the next section, $\bar{m}_{12}=\frac{m_{12}+m_{21}}{2}$, where $m_{k l}$ is the correlation between the latent additive genetic factors underlying phenotype $Y_{k}$ in fathers and phenotype $Y_{l}$ in mothers $(k, l \in\{1,2\})$. Thus, there is positive assortative mating at the additive genetic level for phenotypes $k$ and $l$ if $m_{k l}>0$ and/or $m_{l k}>0$.

The resulting model has 19 free parameters: $\rho_{A}, \rho_{C}$, $\rho_{E}, m_{11}, m_{12}, m_{21}, m_{22}$ and 4 free parameters for each of the matrices a, $\mathbf{c}$ and e. However, only nine moments can be computed from the data: the cross-trait covariance between the MZ twins and, for each trait, the covariance between the MZ twins; the corresponding three moments for the DZ twins; and the population variances of the two traits as well as the population cross-trait covariance. Since the number of parameters exceeds the number of independently informative equations, at least ten identifying assumptions need to be made. In the standard decomposition, it is assumed that $\mathbf{a}, \mathbf{c}$, and $\mathbf{e}$ are diagonal (i.e. that $\left.a_{12}=a_{21}=c_{12}=c_{21}=e_{12}=e_{21}=0\right)$ and that $\Gamma_{M}=0$. Under these assumptions, the remaining parameters are identified. The substantive implication of the diagonality assumption is that, while the latent factors underlying the two traits may be correlated, each latent factor may only influence its respective trait. ${ }^{3}$ The restriction that $\Gamma_{M}=0$ means that there is no assortative mating (including crosstrait assortative mating) for phenotypes $Y_{1}$ and $Y_{2}{ }^{4}$ Below, we also discuss the consequence of assuming different, more realistic values of $\Gamma_{M}$.

Estimates of the shares of the observed variance in either trait attributable to additive genetic factors $\left(a^{2}\right)$, common environment $\left(c^{2}\right)$, and unique environment $\left(e^{2}\right)$ can be obtained from the above parameters. Examination of Eq. 1 and some algebra reveals that

$\operatorname{Var}\left(Y_{k}\right)=\mathbf{E}\left[\left(Y_{k}^{i}\right)^{2}\right]=1=\left(a_{k k}\right)^{2}+\left(c_{k k}\right)^{2}+\left(e_{k k}\right)^{2}$.

We thus see that $a_{k}^{2}=a_{k k}^{2}, c_{k}^{2}=c_{k k}^{2}$, and $e_{k}^{2}=e_{k k}^{2}(k=$ $1,2)$. It also follows from Eq. 1 that the within-individual cross-trait correlation is given by

$$
\begin{aligned}
\operatorname{corr}\left(Y_{1}, Y_{2}\right) & =\mathbf{E}\left[Y_{1}^{i} Y_{2}^{i}\right] \\
& =a_{11} a_{22} \rho_{A}+c_{11} c_{22} \rho_{C}+e_{11} e_{22} \rho_{E} .
\end{aligned}
$$

The shares of the cross-trait phenotypic correlation attributable to additive genetic factors, common environment, and unique environment are thus $\frac{a_{11} a_{22} \rho_{A}}{\operatorname{corr}\left(Y_{1}, Y_{2}\right)}, \frac{c_{11} c_{22} \rho_{C}}{\operatorname{corr}\left(Y_{1}, Y_{2}\right)}$, and $\frac{e_{11} e_{22} \rho_{E}}{\operatorname{corr}\left(Y_{1}, Y_{2}\right)}$, respectively. ${ }^{5}$ These shares depend on the correlations between the latent factors underlying both traits $\left(\rho_{A}\right.$, $\rho_{C}$, and $\rho_{E}$ ) and on the shares of observed phenotypic variance explained by each of the latent factors $\left(a^{2}, c^{2}\right.$, and $\left.e^{2}\right)$.

\section{Assortative mating in the bivariate ACE model}

We now show that assortative mating enters the bivariate ACE model as described in the previous section. For this, it will be useful to augment the model to include individual $i$ 's father and mother. We assume that we are in genetic

\footnotetext{
${ }^{3}$ An alternative way to proceed is to assume that the matrices a, c, and $\mathbf{e}$ are lower triangular (i.e. that $a_{12}=c_{12}=e_{12}=0$ ) and that $\rho_{A}=\rho_{C}=\rho_{E}=0$, while maintaining the assumption that $\Gamma_{M}=0$. The substantive implication of the first assumption is that the latent factors $A_{1}, C_{1}$, and $E_{1}$ of the first trait $Y_{1}$ can affect the second trait $Y_{2}$, whereas the latent factors $A_{2}, C_{2}$, and $E_{2}$ of the second trait $Y_{2}$ cannot affect the first trait $Y_{1}$. The second assumption implies that the latent factors of the two traits are not correlated, even within individuals. The model resulting from this alternative set of assumptions is sometimes referred to as a Cholesky decomposition. That model spans the same space as our preferred model, and it is thus possible to transform the parameters from either model into the parameters of the other (see Loehlin 1996, for a more thorough discussion).

${ }^{4}$ Alternatively, that restriction implies that if there is assortative mating, it does not have consequences at the genetic level. This might be the case, for example, if social homogamy fully explained the spousal phenotypic resemblance.

5 The term "share" in this context can be misleading, as correlations can be negative and these "shares" can therefore be negative.
} 
equilibrium and that all the parameters of the model are fixed across generations. We can write

$$
\begin{aligned}
\mathbf{E}\left[A_{k}^{i} \mid A_{k}^{F_{i}}, A_{k}^{M_{i}}\right] & =\frac{1}{2}\left(A_{k}^{F_{i}}+A_{k}^{M_{i}}\right) \\
\Longrightarrow A_{k}^{i} & =\frac{1}{2}\left(A_{k}^{F_{i}}+A_{k}^{M_{i}}\right)+\epsilon_{k}^{i},
\end{aligned}
$$

where, as above, $A_{k}^{i}$ is the latent additive genetic factor underlying phenotype $Y_{k}^{i}(k=1,2)$ for individual $i ; F_{i}$ and $M_{i}$ refer to $i$ 's father and mother, respectively; and $\epsilon_{k}^{i}$ is an error term independent of the parental genotypes. We rewrite the error term as

$\epsilon_{k}^{i}=\theta_{k} S_{k}^{i}$,

where $\theta_{k}$ is a normalizing constant and $\mathbf{E}\left[\left(S_{k}^{i}\right)^{2}\right]=1$, thus adhering to the convention of working with standardized variables. The expression $\theta_{k} S_{k}^{i}$ is the deviation due to Mendelian segregation (Otto et al. 1994). Finally, we let $\mathbf{E}\left[S_{1}^{i} S_{2}^{i}\right]=\rho_{S}{ }^{6}$ The first equality in Eq. 3 implies that $\mathbf{E}\left[S_{k}^{i} \mid A_{k}^{F_{i}}\right]=\mathbf{E}\left[S_{k}^{i} \mid A_{k}^{M_{i}}\right]=\mathbf{E}\left[S_{k}^{i}\right]=0$, and thus $\mathbf{E}\left[S_{k}^{i} A_{k}^{F_{i}}\right]=$ $\mathbf{E}\left[S_{k}^{i} A_{k}^{M_{i}}\right]=0$. For any pair of full siblings (including DZ twins) $i$ and $j$, it follows that

$$
\begin{aligned}
& \mathbf{E}\left(A_{k}^{i} A_{l}^{j}\right)=\mathbf{E}\left[\left(\frac{1}{2}\left(A_{k}^{F_{i}}+A_{k}^{M_{i}}\right)+\theta_{k} S_{k}^{i}\right)\right.\left.\left(\frac{1}{2}\left(A_{l}^{F j}+A_{l}^{M_{j}}\right)+\theta_{l} S_{l}^{j}\right)\right] \\
&=\frac{1}{4}\left(\mathbf{E}\left[A_{k}^{F_{i}} A_{l}^{F_{j}}\right]+\mathbf{E}\left[A_{k}^{M_{i}} A_{l}^{M_{j}}\right]\right. \\
&\left.\quad+\mathbf{E}\left[A_{k}^{F_{i}} A_{l}^{M_{j}}\right]+\mathbf{E}\left[A_{k}^{M_{i}} A_{l}^{F_{j}}\right]\right) \\
&=\frac{1}{4}\left(\rho_{A, k l}+\rho_{A, k l}+m_{k l}+m_{l k}\right)=\frac{1}{2}\left(\rho_{A, k l}+\bar{m}_{k l}\right),
\end{aligned}
$$

where the second equality holds because $S_{k}^{i}$ and $S_{l}^{j}$ are uncorrelated with each other and with the other variables. ${ }^{7}$ The third equality follows from the fact that $F_{i}=F_{j}$ and $M_{i}=M_{j}$ and from our assumption of genetic equilibrium, which implies that $\mathbf{E}\left[\left(A_{k}^{i}\right)^{2}\right]=1$ is constant across generations and thus that $\mathbf{E}\left[A_{k}^{i} A_{l}^{i}\right]=\operatorname{Cov}\left(A_{k}^{i}, A_{l}^{i}\right)=\rho_{A, k l}$. Here, $\rho_{A, k l}$ is the correlation between the latent additive genetic factors underlying phenotypes $k$ and $l .{ }^{8}$ Therefore, $\mathbf{E}\left[A^{i} A^{j^{\prime}}\right]=\frac{1}{2}\left(\Gamma_{A}+\Gamma_{M}\right)$.

So far, we have only considered assortative mating at the additive genetic level. The parameters of the matrix of

\footnotetext{
${ }^{6}$ In a more realistic model, the distribution of $S^{i}=\left(S_{1}^{i}, S_{2}^{i}\right)^{\prime}$ would vary as a function of $A^{F_{i}}$ and $A^{M_{i}}$. For simplicity and tractability, we do not consider such a model.

$7 S_{k}^{i}$ and $S_{k}^{j}$ are uncorrelated with each other because, conditional on the parents' genomes, the precise genetic draw from one DZ twin does not affect that of the other.

${ }^{8}$ Thus, $\rho_{A, k l}=1$ if $k=l$ and $\rho_{A, k l}=\rho_{A}$ if $k=1$ and $l=2$ as in the previous section. Also, we use $\bar{m}_{k l}$ to denote $m_{k l}$ when $k=l$.
}

genetic assortative mating correlations $\Gamma_{M}$ are not directly observable, so it would be useful to have a mapping relating $\Gamma_{M}$ to observable parameters. Unfortunately, to our knowledge, no one has yet derived such a mapping for the general case of unconstrained multivariate assortative mating. ${ }^{9}$ Gianola $(1982)^{10}$ considers two special cases of interest to the livestock industry. The first case is when assortative mating is actively practiced on one phenotype only and a second phenotype is genetically correlated with the first due to pleiotropy (or linkage) — such as when, for instance, large bulls are mated with large cows, and the second trait is genetically correlated with cattle size. The second case is when mating pairs are assorted to have a certain correlation between phenotype $\mathrm{X}$ in males and phenotype $\mathrm{Y}$ in females-such as when, to use Gianola's example, high milk production females are mated to fast growing males. Assortative mating for height and intelligence in humans is more complex and unlikely to fit either of these cases. We leave the derivation of a general mapping to future research. However, the above discussion should make it clear that it is important to investigate how sensitive the results from the standard bivariate decomposition are to the assumption that $\Gamma_{M}$ is equal to zero for traits with high assortative mating.

We now turn our attention to three additional features of the model which merit further exploration. First, we show that in the augmented model (in which the elements of the $\Gamma_{M}$ matrix are not constrained to be zero), the correlation between two traits can be decomposed into parts attributable to assortative mating, pleiotropy, common environment, and individual environment. Second, we investigate the bias which arises if a standard bivariate ACE model is estimated in the presence assortative mating. Finally, we consider how within-family correlations can be used to shed light on the sources of a phenotypic correlation.

\section{Decomposition of $\rho_{A}$}

Observe that

$$
\begin{aligned}
1 & =\mathbf{E}\left[\left(A_{k}^{i}\right)^{2}\right]=\mathbf{E}\left[\left(\frac{1}{2}\left(A_{k}^{F i}+A_{k}^{M_{i}}\right)+\theta_{k} S_{k}\right)^{2}\right] \\
& =\frac{1}{2}\left(1+m_{k k}\right)+\left(\theta_{k}\right)^{2} \Longrightarrow \theta_{k}=\sqrt{\frac{1-m_{k k}}{2}},(k=1,2),
\end{aligned}
$$

and that

\footnotetext{
${ }^{9}$ Observe that it is not correct to simply assume that $m_{k l}=$ $\sqrt{a_{k k}^{2} a_{l l}^{2}} r_{k l}$, where $r_{k l}$ is the phenotypic spousal correlation for traits $k$ and $l(k, l \in\{1,2\})$. To see, consider Gianola's first case below. There, $\bar{m}_{12}>r_{12}$ because the phenotypic cross-trait correlation follows from the genetic cross-trait correlation, and not the other way around.

${ }^{10}$ We are thankful to Peter Visscher for directing us to this paper.
} 


$$
\begin{aligned}
\rho_{A} & =\mathbf{E}\left[A_{1}^{i} A_{2}^{i}\right] \\
& =\mathbf{E}\left[\left(\frac{1}{2}\left(A_{1}^{F i}+A_{1}^{M_{i}}\right)+\theta_{1} S_{1}\right) \cdot\left(\frac{1}{2}\left(A_{2}^{F i}+A_{2}^{M_{i}}\right)+\theta_{2} S_{2}\right)\right], \\
& =\frac{1}{2}\left(\rho_{A}+\bar{m}_{12}\right)+\theta_{1} \theta_{2} \rho_{S} .
\end{aligned}
$$

It follows that

$$
\rho_{A}=\bar{m}_{12}+\sqrt{\left(1-m_{11}\right)\left(1-m_{22}\right)} \rho_{S} .
$$

Therefore, in equilibrium, the correlation between the latent additive genetic factors underlying phenotypes $Y_{1}$ and $Y_{2}$ is equal to the sum of a term accounting for crosstrait assortative mating and a term accounting for the genetic correlation arising from pleiotropy (or linkage). In the limiting case where there is no assortative mating, $\rho_{A \mid m_{11}=\bar{m}_{12}=m_{22}=0}=\rho_{S}$. Without assortative mating, the genetic correlation must be entirely attributable to pleiotropy (or linkage), and thus $\rho_{s}$ is the genetic correlation that is attributable to pleiotropy (or linkage). We can thus rewrite Eq. 5 as

$$
\rho_{A}=\bar{m}_{12}+\sqrt{\left(1-m_{11}\right)\left(1-m_{22}\right)} \rho_{\text {A,Pleiotropy }}
$$

In the limiting case where there is no pleiotropy, $\rho_{A \mid \rho_{S}=0}=\bar{m}_{12}$ and the genetic correlation is entirely due to cross-trait assortative mating. Substituting Eq. 6 into Eq. 2 yields

$$
\begin{aligned}
& \operatorname{corr}\left(Y_{1}, Y_{2}\right)=a_{11} a_{22} \bar{m}_{12} \\
& \quad+a_{11} a_{22} \sqrt{\left(1-m_{11}\right)\left(1-m_{22}\right)} \rho_{A, \text { Pleiotropy }} \\
& \quad+c_{11} c_{22} \rho_{C}+e_{11} e_{22} \rho_{E} .
\end{aligned}
$$

The correlation between phenotypes $Y_{1}$ and $Y_{2}$ can thus be decomposed into parts attributable to assortative mating, pleiotropy, common environment, and individual environment.
A lower bound for the share of the genetic correlation

Observe that for a pair of MZ twins,

$\mathbf{E}^{M Z}\left[Y_{i} Y_{j}^{\prime}\right]=\mathbf{a} \Gamma_{A} \mathbf{a}^{\prime}+\mathbf{c} \Gamma_{C} \mathbf{c}^{\prime}$,

and that for a pair of DZ twins,

$\mathbf{E}^{D Z}\left[Y_{i} Y_{j}^{\prime}\right]=\frac{1}{2} \mathbf{a}\left(\Gamma_{A}+\Gamma_{M}\right) \mathbf{a}^{\prime}+\mathbf{c} \Gamma_{C} \mathbf{c}^{\prime}$,

implying that

$\mathbf{a} \Gamma_{A} \mathbf{a}^{\prime}=2\left(\mathbf{E}^{M Z}\left[Y^{i} Y^{j \prime}\right]-\mathbf{E}^{D Z}\left[Y^{i} Y^{j^{\prime}}\right]\right)+\mathbf{a} \Gamma_{M} \mathbf{a}^{\prime}$.

Computing the off-diagonal elements of the symmetric matrices on both sides of the above equation gives

$a_{11} a_{22} \rho_{A}=2\left(\mathbf{E}^{M Z}\left[Y_{1}^{i} Y_{2}^{j}\right]-\mathbf{E}^{D Z}\left[Y_{1}^{i} Y_{2}^{j}\right]\right)+a_{11} a_{22} \bar{m}_{12}$.

Interestingly, when there is no cross-trait assortative mating at the genetic level $\left(\bar{m}_{12}=0\right)$, the share of the cross-trait phenotypic correlation attributable to additive genetic factors $\left(\frac{a_{11} a_{22} \rho_{A}}{\operatorname{corr}\left(Y_{1}, Y_{2}\right)}\right)$ does not depend on the sametrait assortative mating genetic correlations $\left(m_{11}\right.$ and $\left.m_{22}\right)$. Thus, under the maintained assumptions of the model, if cross-trait assortative mating is nonnegative at the genetic level $\left(\bar{m}_{12} \geq 0\right)$, estimates from the standard bivariate ACE model still provide a lower bound for the share of the crosstrait phenotypic correlation attributable to additive genetic factors. The cross-trait genetic correlation itself $\left(\rho_{A}\right)$ is however a function of both the cross-trait and same-trait assortative mating genetic correlations, since the coefficients $a_{11}$ and $a_{22}$ depend on the latter.

\section{Within-family analysis}

The within-family correlation between traits $Y_{1}$ and $Y_{2}$ is defined as $\operatorname{corr}_{W F}\left(Y_{1}, Y_{2}\right)=\operatorname{corr}\left(Y_{1}^{i}-Y_{1}^{j}, Y_{2}^{i}-Y_{2}^{j}\right)$, where individuals $i$ and $j$ are either non-twin siblings or DZ twins. Thus,

$$
\begin{aligned}
\operatorname{corr}_{W F}\left(Y_{1}, Y_{2}\right) & =\frac{\mathbf{E}\left[\left(Y_{1}^{i}-Y_{1}^{j}\right)\left(Y_{2}^{i}-Y_{2}^{j}\right)\right]}{\sqrt{\mathbf{E}\left[Y_{1}^{i}-Y_{1}^{j}\right]^{2} \mathbf{E}\left[Y_{2}^{i}-Y_{2}^{j}\right]^{2}}} \\
& =\frac{\mathbf{E}\left[\left(a_{11} A_{1}^{i}+e_{11} E_{1}^{i}-a_{11} A_{1}^{j}-e_{11} E_{1}^{j}\right)\left(a_{22} A_{2}^{i}+e_{22} E_{2}^{i}-a_{22} A_{2}^{j}-e_{22} E_{2}^{j}\right)\right]}{\sqrt{\mathbf{E}\left[a_{11} A_{1}^{i}+e_{11} E_{1}^{i}-a_{11} A_{1}^{j}-e_{11} E_{1}^{j}\right]^{2} \mathbf{E}\left[a_{22} A_{2}^{i}+e_{22} E_{2}^{i}-a_{22} A_{2}^{j}-e_{22} E_{2}^{j}\right]^{2}}} \\
& =\frac{\mathbf{E}\left[a_{11} \theta_{1}\left(S_{1}^{i}-S_{1}^{j}\right) a_{22} \theta_{2}\left(S_{2}^{i}-S_{2}^{j}\right)+e_{11}\left(E_{1}^{i}-E_{1}^{j}\right) e_{22}\left(E_{2}^{i}-E_{2}^{j}\right)\right]}{\sqrt{\mathbf{E}\left[a_{11} \theta_{1}\left(S_{1}^{i}-S_{1}^{j}\right)+e_{11}\left(E_{1}^{i}-E_{1}^{j}\right)\right]^{2} \mathbf{E}\left[a_{22} \theta_{2}\left(S_{2}^{i}-S_{2}^{j}\right)+e_{22}\left(E_{2}^{i}-E_{2}^{j}\right)\right]^{2}}} \\
& =\frac{a_{11} a_{22} \theta_{1} \theta_{2} \rho_{S}+e_{11} e_{22} \rho_{E}}{\sqrt{\left(\left(a_{11} \theta_{1}\right)^{2}+\left(e_{11}\right)^{2}\right) \cdot\left(\left(a_{22} \theta_{2}\right)^{2}+\left(e_{22}\right)^{2}\right)}} .
\end{aligned}
$$


Therefore, cross-trait assortative mating does not affect the within-family correlation. Unless $\rho_{S}$ and $\rho_{E}$ have different signs, the empirical observation that $\operatorname{corr}_{W}$ ${ }_{F}\left(Y_{1}, Y_{2}\right) \cong 0$ would rule out pleiotropy (or linkage) as a source of genetic correlation, thereby suggesting that assortative mating is responsible. Lastly, observe that expression Eq. 8 collapses nicely to $\operatorname{Corr}_{W F}\left(Y_{1}, Y_{2}\right) \cong$ $\rho_{S}=\rho_{A, \text { Pleiotropy }}$ when $e_{11} \cong e_{22} \cong 0$.

\section{Results}

The correlation between height and intelligence is 0.176 (SE 0.015) in our sample, which is similar to what has been previously reported in the literature. The correlation between height and military aptitude is 0.101 (SE 0.016). Estimates (and their asymptotic standard errors) of the parameters in the standard bivariate ACE model without assortative mating are reported in Table 3, separately for the height-intelligence and the height-military aptitude correlations. The analyses were run in MPLUS (Muthén and Muthén 2006), a numerical optimizer often used in behavior genetics. We followed standard practice and used maximum likelihood estimation under the assumption that the phenotypic variables come from a bivariate normal distribution.

Results suggest that $77 \%, 16 \%$ and $7 \%$ of the observed variance in height is attributable to additive genetic, common environmental, and unique environmental factors, respectively. The corresponding figures are $62 \%, 20 \%$, and $18 \%$ for intelligence, and $55 \%, 11 \%$, and $35 \%$ for military

Table 3 Results from the estimation of the bivariate ACE model for height-intelligence and for height-military aptitude

\begin{tabular}{|c|c|c|c|c|c|}
\hline & \multicolumn{2}{|c|}{ Height-intelligence } & \multicolumn{2}{|c|}{ Height-military aptitude } \\
\hline & & Estimate & SE & Estimate & SE \\
\hline \multirow[t]{3}{*}{ Height } & $a_{1}^{2}$ & 0.77 & 0.03 & 0.77 & 0.03 \\
\hline & $c_{1}^{2}$ & 0.16 & 0.04 & 0.16 & 0.04 \\
\hline & $e_{1}^{2}$ & 0.07 & 0.003 & 0.07 & 0.003 \\
\hline \multirow[t]{3}{*}{ Intelligence } & $a_{2}^{2}$ & 0.63 & 0.04 & & \\
\hline & $c_{2}^{2}$ & 0.20 & 0.04 & & \\
\hline & $e_{2}^{2}$ & 0.18 & 0.01 & & \\
\hline Military & $a_{2}^{2}$ & & & 0.55 & 0.05 \\
\hline \multirow[t]{6}{*}{ Aptitude } & $c_{2}^{2}$ & & & 0.11 & 0.04 \\
\hline & $e_{2}^{2}$ & & & 0.35 & 0.01 \\
\hline & $\rho_{A}$ & 0.08 & 0.04 & 0.09 & 0.04 \\
\hline & $\rho_{C}$ & 0.59 & 0.15 & 0.23 & 0.21 \\
\hline & $\rho_{E}$ & 0.15 & 0.03 & 0.09 & 0.03 \\
\hline & $\ln (L)$ & -13332.32 & & -13765.34 & \\
\hline
\end{tabular}

Note: This table shows the estimates from the bivariate ACE model assuming no assortative mating aptitude. Our point estimates also suggest that the correlation between the latent genetic factors underlying the height and intelligence phenotypes $\left(\rho_{A}\right)$ is a rather modest 0.08 , and that the corresponding correlation from the height-military aptitude decomposition is 0.09 . We obtain much larger estimates for the common environmental correlations $\left(\rho_{C}\right): 0.59$ for height-intelligence and 0.23 for height-military aptitude, but we note the fairly low precision of these estimates. Finally, we obtain modest but very significant estimates for the unique environment correlations $\left(\rho_{E}\right)$ for both variance decompositions.

The estimated shares of the phenotypic cross-trait correlations accounted for by the covariation between $A_{1}$ and $A_{2}, \quad C_{1}$ and $C_{2}$, and $E_{1}$ and $E_{2}$ are presented in Table 4, along with their asymptotic standard errors, for both the height-intelligence and the height-military aptitude cases.

These results suggest that $59 \%$ of the height-intelligence correlation is mediated by common environment, that $31 \%$ is due to additive genetic factors, and that $9 \%$ is due to unique environment. The results of the variance decomposition for height and military aptitude suggest that most of the correlation is accounted for by additive genetic factors $(56 \%)$; the estimated share due to common environment appears quite large at $30 \%$, but is not significantly different from zero.

Given the strong evidence for assortative mating for both height and intelligence, it is interesting to ask how sensitive these results are to the assumption that $\Gamma_{M}$ is a zero matrix. Estimates of the spousal phenotypic correlations for height and intelligence are both large and positive. For example, Bouchard and McGue (1981), in a review of the literature, report an average spousal correlation of 0.33 for intelligence, while Silventoinen et al. (2003) and Vandenberg (1972) report spousal correlations in the neighborhood of 0.30 for height. We know of no published estimates of the spousal height-intelligence phenotypic correlation, but data available to us from the Minnesota Twin Family Study suggests that the correlation is around $0.04{ }^{11}$ Though there exists no mapping relating $\Gamma_{M}$ to observable parameters (as we noted above), we hope this information about spousal resemblance can provide some guidance as to what might be plausible parameter values. To have a better idea of the potential significance of the biasing effect of neglecting assortative mating, we estimated our augmented bivariate ACE model for several assumed values of $\Gamma_{M}$. The results of this exercise are reported in Table 5.

\footnotetext{
11 The 0.04 figure is an average of the two cross-trait correlations. The spousal intelligence correlation in this sample is 0.38 and the height correlation is 0.21 . For a description of the sample, see Iacono et al. (2006).
} 
Table 4 Shares of the height-intelligence and height-military aptitude correlations accounted for by the three latent factors

\begin{tabular}{lllll}
\hline $\begin{array}{l}\text { Share of the phenotypic } \\
\text { correlations accounted for by } \ldots\end{array}$ & \multicolumn{2}{l}{ Height-intelligence } & & \multicolumn{2}{l}{ Height-military aptitude } \\
\cline { 2 - 3 } & Estimate & SE & & Estimate \\
\hline A share & 0.31 & 0.13 & 0.56 & 0.26 \\
C share & 0.59 & 0.13 & 0.30 & 0.26 \\
E share & 0.09 & 0.02 & 0.14 \\
\hline
\end{tabular}

Note: This table shows the estimated shares of the height-intelligence and height-military aptitude correlations that are accounted for by additive genetic factors, shared common environmental factors and shared unique environmental factors. The genetic "share" is defined as $\frac{a_{11} a_{22} \rho_{A}}{\operatorname{corr}\left(Y_{1}, Y_{2}\right)}$ and the common environmental and unique environmental shares are defined analogously as $\frac{c_{11} c_{22} \rho_{C}}{\operatorname{corr}\left(Y_{1}, Y_{2}\right)}$ and $\frac{e_{11} e_{22} \rho_{E}}{\operatorname{corr}\left(Y_{1}, Y_{2}\right)}$

Table 5 Parameter estimates for height-intelligence under different assumed values of the $\Gamma_{M}$ matrix

Note: Estimates and their analytical standard errors are reported constraining variance components to be between 0 and 1 and correlation coefficients to have an absolute value less than one. Analytical standard errors are not given in the last column because several of the inequality constraints are binding, rendering inference based on the information matrix unreliable

\begin{tabular}{|c|c|c|c|c|c|}
\hline$\Gamma_{M}=$ & {$\left[\begin{array}{ll}0.10 & 0.02 \\
0.02 & 0.10\end{array}\right]$} & {$\left[\begin{array}{ll}0.15 & 0.04 \\
0.04 & 0.15\end{array}\right]$} & {$\left[\begin{array}{ll}0.20 & 0.06 \\
0.06 & 020\end{array}\right]$} & {$\left[\begin{array}{l}0.25 \\
0.08\end{array}\right.$} & $\begin{array}{l}0.08 \\
0.25\end{array}$ \\
\hline \multirow[t]{2}{*}{$a_{1}^{2}$} & 0.85 & 0.89 & 0.91 & \multicolumn{2}{|l|}{0.94} \\
\hline & 0.04 & 0.03 & 0.03 & \multicolumn{2}{|l|}{-} \\
\hline \multirow[t]{2}{*}{$c_{1}^{2}$} & 0.07 & 0.04 & 0.02 & \multicolumn{2}{|l|}{0.00} \\
\hline & 0.04 & 0.03 & 0.02 & \multicolumn{2}{|l|}{-} \\
\hline \multirow[t]{2}{*}{$e_{1}^{2}$} & 0.07 & 0.07 & 0.07 & \multicolumn{2}{|l|}{0.07} \\
\hline & 0.003 & 0.003 & 0.003 & \multicolumn{2}{|l|}{-} \\
\hline \multirow[t]{2}{*}{$a_{2}^{2}$} & 0.70 & 0.73 & 0.77 & \multicolumn{2}{|l|}{0.83} \\
\hline & 0.04 & 0.04 & 0.04 & \multicolumn{2}{|l|}{-} \\
\hline \multirow[t]{2}{*}{$c_{2}^{2}$} & 0.13 & 0.10 & 0.06 & 0.00 & \\
\hline & 0.04 & 0.04 & 0.04 & - & \\
\hline$e_{2}^{2}$ & 0.18 & 0.18 & 0.18 & 0.18 & \\
\hline & 0.01 & 0.01 & 0.01 & - & \\
\hline A Share & 0.40 & 0.54 & 0.70 & 0.91 & \\
\hline & 0.14 & 0.12 & 0.12 & - & \\
\hline C Share & 0.51 & 0.36 & 0.20 & 0.00 & \\
\hline & 0.13 & 0.12 & 0.121 & - & \\
\hline E Share & 0.09 & 0.09 & 0.09 & 0.10 & \\
\hline & 0.02 & 0.02 & 0.02 & - & \\
\hline
\end{tabular}

These results demonstrate that the point estimates from the standard bivariate ACE model are sensitive to assumptions about the spousal cross-trait genetic correlation. As the values of the off-diagonal parameters and the values of the diagonal parameters of $\Gamma_{M}$ matrix are progressively increased from 0 to 0.08 and from 0.10 to 0.25 , respectively, the value of the genetic share goes from 0.314 to 0.905 and the value of the common environmental share declines from 0.592 to zero. These changes are quite dramatic and illustrate the sensitivity of the model to even small departures from the assumptions on which it is based. Nonetheless, as we demonstrated analytically above, in the presence of positive cross-trait assortative mating, the standard bivariate ACE model still provides a lower bound for the share of the cross-trait phenotypic correlation attributable to additive genetic factors. Table 5 also illustrates another well-known result, namely that as the diagonal elements of the $\Gamma_{M}$ matrix are progressively increased, the estimated heritabilities rise at the expense of the estimated shared environmental variance components.

Our framework also showed how the within-DZ correlation can be used to help shed light on the sources of the phenotypic correlation between height and intelligence. In our sample, the within-family height-intelligence correlation is 0.10 (SE 0.017). The within-family height-military aptitude correlation is 0.09 (SE 0.022).

\section{Discussion}

Our point estimates from the standard bivariate ACE model with zero assortative mating suggest that both environmental and genetic factors are responsible for the heightintelligence and the height-military aptitude correlations. Specifically, they suggest that common environment and additive genetic effects account for $59 \%$ and $31 \%$ of the 
height-intelligence correlation, respectively; the corresponding numbers for the height-military aptitude correlation are $30 \%$ and $56 \%$. Our height-intelligence results are strikingly similar to those of Sundet et al. (2005): applying the standard bivariate ACE model to a sample of Norwegian conscripts, they found that the correlation between the latent common environmental factors which underlie height and intelligence was 0.56 , only marginally lower than our point estimate of 0.59 . They also estimated the heritability of height at 0.76 and the heritability of intelligence at 0.64 , while our own estimates are 0.77 and 0.63 , respectively. ${ }^{12}$

This finding contrasts with those reported by Silventoinen et al. (2006) who found that for all cohorts in which there was an association between height and intelligence, the association was explained entirely by additive genetic correlation. One possible reason for the discrepancy between our results and those reported by Silventoinen et al. (2006) is the difference between the samples. Silventoinen et al. (2006) perform independent analyses on four cohorts of Dutch twins ranging in age from children to middle-age adults and in size from 156 to 567 twin pairs.

However, a more likely explanation is that Silventoinen et al. (2006) use a different model selection procedure. Procedurally, they do the following: (1) for each cohort, they begin by fitting univariate models to the data for both height and intelligence; (2) they compare the $\chi^{2}$-goodnessof-fit statistics of the models and select the best-fitting model for each trait; and (3) they use those best-fitting models for all subsequent analyses. They find that the univariate $\mathrm{AE}$ model offers adequate fit in every cohort for height and in almost every cohort for intelligence. Thus, in their bivariate model, they only include latent factors for additive genetic effects and for unique environment for height for every cohort, and they do likewise for intelligence in almost every cohort. As a result, there is no $\rho_{C}$ parameter in any of their bivariate models. Moreover, it appears that they drop the $\rho_{E}$ parameter from their model whenever this improves the fit. In other words, they decompose the height-intelligence correlation either into additive genetic and individual environmental effects, or uniquely into additive genetic effects. Dropping $\rho_{C}$ the most important component of the height-intelligence correlation in both in Sundet et al.'s (2005) data and the results reported here-from their model, and sometimes also dropping $\rho_{E}$, quite mechanically leads to the conclusion that $\rho_{A}$ is an important source of the correlation.Therefore, the confidence intervals presented by Silventoinen et al. (2006) do not reflect the uncertainty that stems from the model-selection procedure that is

\footnotetext{
12 These estimates of the heritability of height are lower than the consensus in the literature (Mittler 1971; Silventoinen et al. 2004).
}

employed. It is not clear to us why the fact that a parameter is not statistically significant should justify the restriction that the parameter is equal to zero. This point is further elaborated on by Goldberger (2002).

We also derived the bivariate ACE model for the more general and realistic case where there is assortative mating at the genetic level. Sundet et al. (2005, p. 310) and Silventoinen et al. (2006, p. 587) note that their estimates may be biased if there is assortative mating for the studied traits. Our analysis demonstrates the sensitivity of the estimates from the bivariate ACE models to one of several problematic assumptions. Specifically, changing the assumed values of the cross-trait and same-trait spousal genetic assortative mating correlations from 0 to 0.08 and from 0.10 to 0.25 , respectively, raises the estimated share of the height-intelligence correlation mediated by genes from 0.314 to 0.905 . Given that there is ample uncertainty about the relationship between the elements of $\Gamma_{M}$ and the spousal trait and cross-trait correlations, this further reinforces our conclusion that for traits with assortative mating, bivariate ACE estimate must be approached with caution.

It is important to emphasize that we have only explored one dimension of the model selection uncertainty in multivariate behavior genetic models. The interpretation would be further complicated by attempts to allow for the latent factors to be correlated or for gene action to be non-additive, questions which we have not investigated here. As is well known, information on MZ and DZ twins alone is not sufficient to estimate models with latent factors for additive genetic effects, non-additive genetic effects, common environment, and individual environment. Since the twin model suffers from parameter indeterminacy when both dominance and common environmental effects are present (Keller and Coventry 2005), we suggest that future work use extended twin family samples with data on twins' relatives, thus allowing richer models to be identified. Also, rapid advances in molecular genetics may soon make it possible to study the sources of covariation between traits from a new angle.

Despite our reservations, we believe that our framework provides valuable insights into what could plausibly be learned from studies of this kind. For example, Jensen (1980) notes that the observation that the within-family height-intelligence correlation is small and only marginally significant, in contrast to the between-family correlation, is consistent with the hypothesis that the heightintelligence correlation stems from assortative mating. This reasoning is consistent with expression Eq. 8. Johnson (1991), on the other hand, in a review of the literature, concludes that there is a marginally significant positive within-family height-intelligence correlation, and that this suggests that pleiotropy plays some role in the heightintelligence correlation. We also report a significant 
positive within-family height-intelligence correlation, adding further weight to the hypothesis that pleiotropy is at play. Though expression Eq. 8 shows that this within-family correlation could in part be the result of a correlation between the latent unique environment factors underlying height and intelligence rather than of pleiotropy, the conclusion that pleiotropy cannot account for part of the height-intelligence correlation appears to be premature.

Previous research provides little insight into the genetic mechanisms responsible for the association between height and various aspects of cognition. The difficulty of pinpointing specific mechanisms is partly due to the fact that both height and intelligence are polygenic traits. While a number of promising markers for height have been identified, they only explain a small share of the heritable variation (Weedon and Frayling 2008). As for decoding the molecular genetic structure underlying intelligence, an extraordinarily complex trait, the problems appear to be even less tractable (Butcher et al. 2007; Deary et al. 2009). However, with the advent of genome-wide association technology, we are hopeful that key genetic markers will be identified resulting in a greater understanding of the genetics of the association between height and various aspects of cognition.

\section{Concluding remark}

We developed a general theoretical framework to study the correlation between two traits. We used this framework to extend the standard bivariate ACE model to account for assortative mating and to derive formulas to decompose a cross-trait genetic correlation into components attributable to assortative mating and pleiotropy and to decompose a cross-trait within-family correlation. Our results from the standard bivariate ACE model without assortative mating suggest that common environment explains most of the height-intelligence correlation. However, we caution that our estimates from the standard bivariate ACE model are sensitive to assumptions about assortative mating. In fact, we show that assuming more plausible values for the matrix of assortative mating correlations $\Gamma_{M}$ dramatically changes the results of the height-intelligence decomposition, implying that the correlation is primarily mediated by genetic factors rather than by common environment. Also, although we use the largest sample to date for this kind of analysis, and although our data comes from standardized measurements of male conscripts in a tight age range, our estimates are not very precise, as reflected by the size of the standard errors. This leads us to emphasize the difficulty of disentangling the sources of covariation between two traits with samples consisting only of MZ and DZ twins. Future work could benefit from the use of large extended twin family samples. Alternatively, given the rapid pace at which knowledge about the genome is increasing, it may be that such questions will soon be more easily tackled through an approach that seeks to understand how specific genes directly affect the phenotypes of interest.

Acknowledgements We are grateful to Bill Dickens, James Lee, Joe Rodgers, Peter Visscher and two anonymous reviewers for helpful comments. We are also grateful to The Jan Wallander and Tom Hedelis Foundation, the Swedish Research Council, and the Swedish Council for Working Life and Social Research for financial support. Jonathan Beauchamp also thanks the Trudeau Foundation and the Social Sciences and Humanities Research Council of Canada for financial support.

Open Access This article is distributed under the terms of the Creative Commons Attribution Noncommercial License which permits any noncommercial use, distribution, and reproduction in any medium, provided the original author(s) and source are credited.

\section{References}

Abbott RD, White LR, Ross GW, Petrovitch H, Masaki KH, Snowdon DA, Curb JD (1998) Height as a marker of childhood development and late-life cognitive function: the Honolulu-Asia Aging Study. Pediatrics 102:602-609

Bouchard TJ Jr, McGue M (1981) Genetic and environmental influences on human psychological differences. Science 212: $1055-1059$

Butcher LM, Davis OS, Craig IW, Plomin R (2007) Genome-wide quantitative trait locus association scan of general cognitive ability using pooled DNA and $500 \mathrm{~K}$ single nucleotide polymorphism microarrays. Genes Brain Behav 7:435-446

Carlstedt B (2000) Cognitive abilities: aspects of structure, process and measurement. $\mathrm{PhD}$ dissertation at the Department of Psychology, Gothenburg University Göteborg: Acta Universitatis Gothoburgensis

Deary IJ, Johnson W, Houlihan LM (2009) Genetic foundations of human intelligence. Hum Genet 126:215-232

Eaves LJ, Heath AC (1981) Detection of the effects of asymmetric assortative mating. Nature 282:205-206

Eaves LJ, Last KA, Young PA, Martin NG (1978) Model-fitting approaches to the analysis of human behavior. Heredity 41:249320

Eaves LJ, Heath AC, Martin NG (1984) A note on the generalized effects of assortative mating. Behav Genet 14:371-376

Eide MG, Øyen N, Skjærven R, Nilsen ST, Bjerkedal T, Tell GS (2005) Size at birth and gestational age as predictors of adult height and weight. Epidemiology 16:175-181

Falconer DS, Mackay TFC (1981) Introduction to quantitative genetics. Longman, New York

Gianola D (1982) Assortative mating and the genetic correlation. Theor Appl Genet 62:225-231

Goldberger AS (2002) Structural equation models in human behavior genetics. Wisonsin Madison-Social Systems Working Papers 22

Gomez-Pinilla F (2008) The influences of diet and exercise on mental health through hormesis. Ageing Res Rev 7:49-62

Humphreys LG, Davey TC, Park RK (1985) Longitudinal correlation analysis of standing height and intelligence. Child Dev 56:14651478

Iacono WG, McGue M, Krueger RF (2006) Minnesota center for twin and family research. Twin Res Hum Genet 978-984:307-311 
Isacsson G (2004) Estimating the economic return to educational levels using data on twins. J Appl Econ 19:99-119

Jensen AR (1980) Uses of sibling data in educational and psychological research. Am Educ Res J 17:153-170

Johnson FW (1991) Biological factors and psychometric intelligence: A review. Genet Soc Gen Psychol Monogr 117:313-357

Kanazawa S, Reyniers DJ (2009) The role of height in the sex difference in intelligence. Am J Psychol 122: 527-536

Keller MC, Coventry WL (2005) Quantifying and addressing parameter indeterminacy in the classical twin design. Twin Res Hum Genet 8:201-213

Keller MC, Medland SE, Duncan LE, Hatemi PK, Neale MC, Maes HHM, Eaves LJ (2009) Modeling extended twin family data I: description of the Cascade model. Twin Res Hum Genet 12:8-18

Lichtenstein P, Sullivan PF, Cnattingius S, Gatz M, Johansson S, Carlström E, Björk C, Svartengren M, Volk A, Klareskog L, de Faire U, Schalling M, Palmgren J, Pedersen NL (2006) The Swedish twin registry in the third millennium: an update. Twin Res Hum Genet 9:875-882

Lilieblad B, Ståhlberg B (1977) Reliabilitet hos psykologiska bedö mningar vid inskrivningsprövning. FOA-rapport C 55011-H7

Lindqvist E, Vestman R (forthcoming) The labor market returns to cognitive and noncognitive ability: evidence from the Swedish enlistment. Am Ec J Appl

Loehlin JC (1996) The Cholesky approach: a cautionary note. Behav Genet 26:65-69

Martin NG, Eaves LJ, Heath AC, Jardine R, Feingold LM, Eysenck HJ (1986) Transmission of social attitudes. Proc Natl Acad Sci USA 83:4364-4369

Martyn CN, Gale CR, Sayer AA, Fall C (1996) Growth in utero and cognitive function in adult life: follow up study of people born between 1920 and 1943. Brit Med J 312:1393-1396

Mittler P (1971) The study of twins. Harmondsworth, Penguin

Muthén LK, Muthén BO (2006) Mplus statistical analysis with latent variables users guide. Version 41. Los Angeles, CA

Neale MC, Cardon LR (1992) Methodology for genetic studies of twins and families. Kluwer Academic Publishers, North Holland

Neisser U, Boodoo G, Bouchard TJ Jr, Boykin AW, Brody N, Ceci SJ, Halpern DF, Loehlin JC, Perlo R, Sternberg RJ, Urbina S (1996) Intelligence: knowns and unknowns. Am Psychol 51:77-101

Otto SP, Christiansen FB, Feldman MW (1994) Genetic and cultural inheritance of continuous traits. Morrison Institute for Population and Resource Studies Working Paper
Posthuma D, de Geus EJ, Neale MC, Hulsho Pol HE, Baare WEC, Kahn RS, Boomsma D (2000) Multivariate genetic analysis of brain structure in an extended twin design. Behav Genet 30:311-319

Silventoinen K, Sammalisto S, Perola M, Boomsma DI, Cornes BK, Davis C, Dunkel L, de Lange M, Harris JR, Hjelmborg JVB, Luciano M, Martin NG, Mortensen J, Nisticò L, Pederesen NL, Skytte A, Spector TD, Stazi AM, Willemsen G, Jaakko K (2003) Heritability of adult body height: a comparative study of twin cohorts in eight countries. Twin Res Hum Genet 6:399-408

Silventoinen K, Krueger RF, Bouchard TJ Jr, Kaprio J, McGue M (2004) Heritability of body height and educational attainment in an international context: comparison of adult twins in Minnesota and Finland. Am J Hum Biol 16:544-555

Silventoinen K, Posthuma D, van Beijsterveldt $\mathrm{T}$, Bartels $\mathrm{M}$, Boomsma DI (2006) Genetic contributions to the association between height and intelligence: evidence from Dutch twin data from childhood to middle age. Genes Brain Behav 5:585-595

Steckel R (1995) Stature and the standard of living. J Econ Lit 33:1903-1940

Sundet JM, Tambs K (2005) Resolving the genetic and environmental sources of the correlation between height and intelligence: a study of nearly 2600 Norwegian male twin pairs. Twin Res Hum Genet 8:307-311

Teasdale TW, Sørensen TIA, Owen DR (1989) Fall in association of height with intelligence and educational level. Brit Med J 298:1292-1293

Tuvemo T, Jonsson B, Persson I (1999) Intellectual and physical performance and morbidity in relation to height in a cohort of 18-year-old Swedish conscripts. Horm Res 52:186-191

van Dam PS, de Winter CF, de Vries R, van der Grond J, Drent ML, Lijffijt M, Kenemans LJ, Aleman A, de Haan EHF, Koppeschaar HPF (2005) Childhood-onset growth hormone deficiency cognitive function and brain $\mathrm{N}$-acetylaspartate. Psychoneuroendocrinology 30:357-363

Vandenberg SG (1972) Assortative mating or who marries whom? Behav Genet 2:127-157

Weedon MN, Frayling TM (2008) Reaching new heights: insights into the genetics of human stature. Trends Genet 24:595-603

Wheeler PG, Bresnahan K, Shephard BA, Lau J, Balk EM (2004) Short stature and functional impairment: a systematic review. Arch Pediatr Adolesc Med 158:236-243 